\title{
Solutions to a phase-field model of sea ice growth
}

\section{Yangxin Tang ${ }^{1 *}$}

${ }^{\text {"Correspondence: }}$ tangyangxin@i.shu.edu.cn

'Department of Mathematics, Shanghai University, Shanghai, P.R. China

\section{Springer}

\begin{abstract}
We shall apply the phase-field method to investigate the dynamics of sea ice growth. The model consists of two parabolic equations. The existence and uniqueness of weak solutions to an initial-boundary value problem of this model is proved. Then the regularity, large-time behavior of solutions are studied, also the existence of global attractor is proved. The main technique in this article is energy method. Our existence proof is only valid in one space dimension.
\end{abstract}

MSC: $35 \mathrm{~K} 51 ; 74 \mathrm{~N} 20$

Keywords: Phase-field model; Sea ice; Weak solution; Global attractor; Large time behavior

\section{Introduction}

Due to global warming, which leads to significant climate changes and more and more frequently occurring severe weather disasters, the study of global warming seems more important than ever since sea ice has begun to melt (see, e.g., [1-3]) and this makes sea level rise considerably so that some islandish countries may vanish. In this paper we shall employ a phase-field approach to model the growth of sea ice. This approach, though it has been developed since 1980s, thus is very young, is now a very powerful tool for both theoretical and numerical studies in many fields (see, e.g., [4, 5]). To our knowledge, the application of a two-phase field model to investigation of sea ice growth presented in this paper is the first one in phase-field modeling for sea ice evolution.

The evolution of macroscopic sea ice has been studied by means of the classic Stefan's problem (see, e.g., [6]). Fluid flow through sea ice is another point of interest. The permeability of sea ice is important in many physical processes such as the melting and draining from sea ice surface during the melting season (see, e.g., [7]). A new simple model which includes turbulent transport of heat and salt between ice and ocean is introduced and solved analytically (see, e.g., [8]). The mesoscopic numerical simulation of sea ice crystals growth has been studied through Voronoi dynamics during the freezing season (see, e.g., [9]). Overall, sea ice interacts with the climate system of the polar. A one-dimensional enthalpy-based model of sea ice allows for quantitative studies of sea ice and its interaction (see, e.g., [10]). These references need to add appropriate conditions at the interface of tracking movement. Theoretical analysis and numerical simulation are very difficult. In this paper we study a phase-field model for the evolution of the phase interface region in

(c) The Author(s) 2019. This article is distributed under the terms of the Creative Commons Attribution 4.0 International License (http://creativecommons.org/licenses/by/4.0/), which permits unrestricted use, distribution, and reproduction in any medium, provided you give appropriate credit to the original author(s) and the source, provide a link to the Creative Commons license, and indicate if changes were made. 
sea water-ice interface phase change problems, which was derived in [5]. The author only simulated dendritic crystal growth without theoretical analysis. We will use the phasefield method in the sea ice growth, more precisely, I do theoretical analysis, regularities, and large time behavior. We formulate this initial-boundary value problem in the onedimensional case and conclude the introduction by stating our main result.

Let $\Omega \subset \mathbb{R}^{3}$ be an open set. We introduce a phase-field variable (the order parameter $p \in \mathbb{R})$ to represent the physical state of the system in time and space, that is, to distinguish the liquid phase and solid phase, such as the solid state when the variable is 1 . The liquid phase is expressed when the variable is 0 . We restrict ourselves to that type of order parameter $p$, which describes the evolution of phase interfacial region.

Now let us establish the free energy function $F$ of the system based on the order parameter $p$, their spatial derivatives $\nabla p$, and the local temperature:

$$
F[p, T]=\int_{\Omega}\left(\frac{\varepsilon_{12}^{2}}{2}|\nabla p|^{2}+\widehat{\psi}(p)+e_{0} T^{2}\right) d V .
$$

Setting

$$
\widehat{\psi}(p)=\frac{1}{4 a_{12}}\left(p^{2}(1-p)^{2}-m_{12}^{2}\left(\frac{1}{3} p^{3}+p^{2}(1-p)-\frac{1}{3}(1-p)^{3}-(1-p)^{2} p\right)\right),
$$

we choose for $\widehat{\psi} \in C^{2}(\mathbb{R},[0, \infty))$ a direct extension of the double well potential with minima at $p=0$ and $p=1$. Here, $e_{0}, \varepsilon_{12}, a_{12}, m_{12}$ are thermophysical data.

$T$ is temperature, it satisfies

$$
T=\frac{e}{e_{0}}+\frac{1}{2} h(p)
$$

where $h(p)$ is a non-decreasing smooth function satisfying $h(0)=0$ near $p=0$ and $h(1)=1$ near $p=1, e$ is the local enthalpy, and $e_{0}$ satisfies

$$
e_{0}=\frac{L^{2}}{T_{M} c_{p^{*}}}
$$

$L$ is the latent heat of fusion for sea-water, $T_{M}$ is the melting temperature, and $c_{p^{*}}$ is the specific heat of sea water.

For the two-phase case, we get the following systems:

$$
\begin{aligned}
\frac{\partial p}{\partial t} & =\frac{1}{\tau_{12}}\left(\varepsilon_{12}^{2} \Delta p-\frac{1}{2 a_{12}} p(1-p)(1-2 p)+\frac{m_{12}}{a_{12}} p(1-p)\right)-\kappa T \frac{\partial h}{\partial p}, \\
\frac{\partial T}{\partial t} & =\nabla \cdot(D \nabla T)+\frac{1}{2} \frac{\partial h}{\partial p} \frac{\partial p}{\partial t}
\end{aligned}
$$

for $(t, x) \in(0, \infty) \times \Omega, \kappa, D$ are constants. The boundary and initial conditions are

$$
\begin{aligned}
& p(t, x)=0, \quad(t, x) \in[0, \infty) \times \partial \Omega, \\
& T(t, x)=0, \quad(t, x) \in[0, \infty) \times \partial \Omega, \\
& p(0, x)=p_{0}(x), \quad x \in \Omega, \\
& T(0, x)=T_{0}(x), \quad x \in \Omega .
\end{aligned}
$$


Now we make some assumptions. We assume that all functions depend on the variables $x_{1}$ and $t$ and, to simplify the notation, denote $x_{1}$ by $x$. The set $\Omega$ is a bounded open interval. We write $Q_{T_{e}}:=\left(0, T_{e}\right) \times \Omega$, where $T_{e}$ is a positive constant, and define

$$
(v, \varphi)_{\mathbb{Z}}=\int_{\mathbb{Z}} v(y) \varphi(y) d y
$$

for $\mathbb{Z}=\Omega$ or $\mathbb{Z}=Q_{T_{e}}$.

Then, under these assumptions, equations (1.2)-(1.3) in the case of one dimension can be rewritten as follows:

$$
\begin{aligned}
p_{t}= & \frac{1}{\tau_{12}}\left(\varepsilon_{12}^{2} p_{x x}-\frac{1}{2 a_{12}} p(1-p)(1-2 p)+\frac{m_{12}}{a_{12}} p(1-p)\right) \\
& -\kappa T \frac{\partial h}{\partial p}, \quad \text { in }(t, x) \in\left(0, T_{e}\right) \times \Omega \\
T_{t}= & D T_{x x}+\frac{1}{2} \frac{\partial h}{\partial p} \frac{\partial p}{\partial t}, \quad \text { in }(t, x) \in\left(0, T_{e}\right) \times \Omega,
\end{aligned}
$$

where $\kappa=\frac{2 e_{0}}{\tau_{12}}$. The boundary and initial conditions therefore are

$$
\begin{aligned}
& p(t, x)=0, \quad \text { on }(t, x) \in\left[0, T_{e}\right] \times \partial \Omega, \\
& T(t, x)=0, \quad \text { on }(t, x) \in\left[0, T_{e}\right] \times \partial \Omega, \\
& p(0, x)=p_{0}(x), \quad \text { in } x \in \Omega, \\
& T(0, x)=T_{0}(x), \quad \text { in } x \in \Omega .
\end{aligned}
$$

Definition 1.1 Let $p_{0} \in H_{0}^{1}(\Omega), T_{0} \in L^{2}(\Omega)$. A function $(p, T)$ with

$$
\begin{aligned}
& p \in L^{\infty}\left(0, T_{e} ; H_{0}^{1}(\Omega)\right) \cap L^{2}\left(0, T_{e} ; H^{2}(\Omega)\right), \\
& T \in L^{\infty}\left(0, T_{e} ; L^{2}(\Omega)\right) \cap L^{2}\left(0, T_{e} ; H_{0}^{1}(\Omega)\right),
\end{aligned}
$$

is a weak solution to problem (1.8)-(1.13) if, for all $\varphi \in C_{0}^{\infty}\left(\left(-\infty, T_{e}\right) \times \Omega\right)$, there hold

$$
\begin{aligned}
0= & \left(p, \varphi_{t}\right)_{Q_{T_{e}}}-\frac{1}{\tau_{12}} \varepsilon_{12}^{2}\left(p_{x}, \varphi_{x}\right)_{Q_{T_{e}}}-\frac{1}{\tau_{12}} \frac{1}{2 a_{12}}(p(1-p)(1-2 p), \varphi)_{Q_{T_{e}}} \\
& +\frac{1}{\tau_{12}} \frac{m_{12}}{a_{12}}(p(1-p), \varphi)_{Q_{T_{e}}}-\kappa T\left(\frac{\partial h}{\partial p}, \varphi\right)_{Q_{T_{e}}}+\left(p_{0}, \varphi(0)\right)_{\Omega}, \\
0= & \left(T, \varphi_{t}\right)_{Q_{T_{e}}}-\left(D T_{x}, \varphi_{x}\right)_{Q_{T_{e}}}+\frac{1}{2}\left(\frac{\partial h}{\partial p}, \varphi\right)_{Q_{T_{e}}}+\left(T_{0}, \varphi(0)\right)_{\Omega} .
\end{aligned}
$$

The main results of this article are as follows.

Theorem 1.1 For all $p_{0} \in H_{0}^{1}(\Omega)$ and $T_{0} \in L^{2}(\Omega)$, there exists a unique weak solution $(p, T)$ of problem (1.8)-(1.13), which in addition to (1.14)-(1.15) satisfies

$$
p_{t} \in L^{2}\left(Q_{T_{e}}\right), \quad p \in L^{4}\left(Q_{T_{e}}\right), \quad T_{t} \in L^{2}\left(0, T_{e} ; H^{-1}(\Omega)\right) .
$$


Theorem 1.2 Assume that $p_{0} \in H^{2}(\Omega), T_{0} \in H_{0}^{1}(\Omega)$, then there exists a weak solution $(p, T)$ of problem (1.8)-(1.13), which in addition to (1.14)-(1.15) satisfies

$$
\begin{aligned}
& p \in L^{\infty}\left(0, T_{e} ; H^{2}(\Omega)\right), \quad p_{t} \in L^{\infty}\left(0, T_{e} ; L^{2}(\Omega)\right) \cap L^{2}\left(0, T_{e} ; H_{0}^{1}(\Omega)\right), \\
& p_{t t} \in L^{2}\left(0, T_{e} ; H^{-1}(\Omega)\right), \\
& T \in L^{2}\left(0, T_{e} ; H^{2}(\Omega)\right) \cap L^{\infty}\left(0, T_{e} ; H_{0}^{1}(\Omega)\right), \quad T_{t} \in L^{2}\left(0, T_{e} ; H^{-1}(\Omega)\right) .
\end{aligned}
$$

Remark Assume that $p_{0} \in H^{k}(\Omega), T_{0} \in H^{k-1}(\Omega)$, regularity will continue to improve, when $k$ is sufficiently large, then weak solution becomes the classical solution.

Definition 1.2 Let $X$ be a Banach space. A one-parameter family $S(t), 0 \leq t<\infty$, of bounded linear operators from $X$ into $X$ is a semigroup bounded linear operator on $X$ if

(i) $S(0)=I(I$ is an identity operator on $X)$,

(ii) $S(s+t)=S(s) S(t)$ for every $t, s \geq 0$ (the semigroup property).

Theorem 1.3 Let $\Omega$ denote an open bounded set of $\mathbb{R}$ and $g_{1}$ denote a polynomial. The semigroup $p(t)$ associated with the initial-boundary-value problem (1.8)-(1.13) possesses a maximal attractor $A$ which is bounded in $H_{0}^{1}(\Omega)$, compact and connected in $L^{2}(\Omega)$. Its basin of attraction is the whole space $L^{2}(\Omega)$, A attracts the bounded sets of $L^{2}(\Omega)$. Assume that the coefficient is suitably large. Then $\|p\|_{L^{\infty}(\Omega)}$ and $\|T\|^{2}$ decrease exponentially to 0 as $t \rightarrow \infty$.

Notation Let $\Omega$ be a domain in $\mathbb{R}^{n}$, and let $r$ be a positive real number. We denote by $L^{p}(\Omega)$ the class of all measurable functions $u$ defined on $\Omega$ for which

$$
\int_{\Omega}|u(x)|^{r} d x<\infty
$$

The Sobolev space $W^{k, r}(\Omega)$ is defined by

$$
W^{k, r}(\Omega)=\left\{u \in L^{r}(\Omega): D^{\alpha} u \in L^{r}(\Omega), 0 \leq|\alpha| \leq k, 1 \leq r \leq \infty\right\}
$$

where $k$ is any positive integer and $D^{\alpha} u$ is the weak partial derivative. $\|\cdot\|,|\cdot|, C, \mathcal{A}, S(t)$ denote $L^{2}(\Omega)$-norm, the absolute value, various constants, attractor, semigroup, respectively; $\partial_{t}$ or $\frac{d}{d t}$ or a subscript $t$ and $\partial_{x}$ or a subscript $x$ denote the derivative with respect to $t$ and $x$ in the distribution sense, respectively.

The remaining sections are devoted to the proof of Theorem 1.1, Theorem 1.2, and Theorem 1.3. In order to obtain the local solution of the initial-boundary value problem for nonlinear equations (1.8)-(1.13), we construct the approximate sequence

$$
\left\{(p, T)_{n}(t, x)=\left(p^{n}, T^{n}\right)(t, x)\right\}_{n=3}^{\infty} .
$$

We prove the existence of weak solutions by iterative method: Choose a known approximate solution $p^{n-1}, T^{n-1}$ and determine the next $p^{n}, T^{n}$ by solving equations to (1.8)-(1.9), 
proving existence solution by using of Banach's fixed point theorem. When we regard the term $p^{n}$ in (1.9) as known by use of solution of (1.8), a solution will be obtained if convergence of this procedure can be shown. In Sect. 2 we shall establish some a priori estimates for the solution.

We will discuss the regularity of our weak solutions $p, T$ for the parabolic systems in Sect. 3.

Section 4 is devoted to investigation of the large time behavior of a solution by using the a priori estimates.

\section{A priori estimates}

In this section we establish a priori estimates for solutions $(p, T)$ to the initial-boundary value problem (1.8)-(1.13).

Lemma 2.1 There holds, for any $t \in\left[0, T_{e}\right]$,

$$
\int_{0}^{t}\left\|p_{t}\right\|^{2} d \tau+\kappa D \int_{0}^{t}\left\|T_{x}\right\|^{2} d \tau+\|T(t)\|^{2}+\left\|p_{x}(t)\right\|^{2}+\|p(t)\|_{L^{4}(\Omega)}^{4} \leq C .
$$

Proof Multiplying (1.8), (1.9) by $p_{t}, 2 \kappa T$ and integrating by parts with respect to $x \in \Omega$,

$$
\begin{aligned}
\frac{1}{2} & \frac{d}{d t}\|T\|^{2}+\frac{\varepsilon_{12}}{\tau_{12}} \frac{d}{d t}\left\|p_{x}\right\|^{2}+2 \kappa D\left\|T_{x}\right\|^{2}+\frac{1}{2 \tau_{12} a_{12}} \int_{\Omega} p(1-p)(1-2 p) p_{t} d x \\
& -\frac{m_{12}}{\tau_{12} a_{12}} \int_{\Omega} p(1-p) p_{t} d x=0 .
\end{aligned}
$$

Integrating (2.2) in $\tau \in[0, t]$, we have

$$
\begin{aligned}
& \int_{0}^{t}\left\|p_{t}\right\|^{2} d \tau+4 \kappa D \int_{0}^{t}\left\|T_{x}\right\|^{2} d \tau+2\|T(t)\|^{2}+\frac{\varepsilon_{12}}{\tau_{12}}\left\|p_{x}(t)\right\|^{2}+c\|p(t)\|_{L^{4}(\Omega)}^{4} \\
& \quad \leq \frac{\varepsilon_{12}}{\tau_{12}}\left\|p_{0 x}\right\|^{2}+2\left\|T_{0}\right\|^{2}+C\left(\left\|p_{0}\right\|_{L^{4}(\Omega)}^{4}+C\right) \leq C .
\end{aligned}
$$

Thus we obtain $p_{t} \in L^{2}\left(Q_{t}\right), p \in L^{\infty}\left(0, t ; H_{0}^{1}(\Omega)\right) \cap L^{4}\left(Q_{t}\right), T \in L^{\infty}\left(0, t ; L^{2}(\Omega)\right) \cap L^{2}(0, t$; $H_{0}^{1}(\Omega)$ ).

Lemma 2.2 There holds, for any $t \in\left[0, T_{e}\right]$,

$$
\begin{aligned}
& \int_{0}^{t}\|p(t)\|_{L^{4}(\Omega)}^{4} d \tau \leq C, \\
& \int_{0}^{t}\left\|T_{t}\right\|_{H^{-1}(\Omega)}^{2} d \tau \leq C .
\end{aligned}
$$

Proof Noting that $\left.f\right|_{\partial \Omega}=0$, we have the Gagliardo-Nirenberg inequality in the form

$$
\|f\|_{L^{4}(\Omega)} \leq c\left\|f_{x}\right\|_{L^{2}(\Omega)}^{\frac{1}{4}}\|f\|_{L^{2}(\Omega)}^{\frac{3}{4}} .
$$

We have

$$
\int_{0}^{t}\|p(\tau)\|^{4} d \tau \leq C\|p(t)\|_{L^{\infty}\left(0, t ; L^{2}(\Omega)\right)}^{3} \int_{0}^{t}\left\|p_{x}(\tau)\right\| d \tau \leq C t^{\frac{1}{2}} \leq C_{t} .
$$


Using equations (1.8), (1.9), we have

$$
\begin{aligned}
\frac{1}{\tau_{12}} \varepsilon_{12}^{2} p_{x x} & =p_{t}+\kappa T \frac{\partial h}{\partial p}-\frac{1}{\tau_{12}}\left(-\frac{1}{2 a_{12}} p(1-p)(1-2 p)+\frac{m_{12}}{a_{12}} p(1-p)\right) \\
& =f_{1} \\
T_{t}=D T_{x x} & +\frac{1}{2} \frac{\partial h}{\partial p} \frac{\partial p}{\partial t} .
\end{aligned}
$$

Taking the $L^{2}(\Omega)$-norm on both sides of equation (2.7), squaring and integrating it in $\tau \in$ $(0, t)$, using relation $(2.1)$, we have

$$
\begin{aligned}
\frac{1}{\tau_{12}} \varepsilon_{12}^{2} \int_{0}^{t}\left\|p_{x x}\right\|^{2} d \tau \leq & C\left(\int_{0}^{t}\left\|p_{t}\right\|^{2} d \tau+\int_{0}^{t}\|T\|^{2}\left\|\frac{\partial h}{\partial p}\right\|_{L^{\infty}(\Omega)}^{2}\right. \\
& \left.+\frac{1}{\tau_{12}}\left(\left\|\frac{1}{2 a_{12}} p(1-p)(1-2 p)\right\|^{2}+\frac{m_{12}}{a_{12}}\|p(1-p)\|^{2}\right)\right) d \tau \leq C .
\end{aligned}
$$

Next we invoke the inequality

$$
\beta\|p\|_{H^{2}(\Omega)}^{2} \leq\left\|f_{1}\right\|+\gamma\|p\|^{2} \quad\left(p \in H^{2}(\Omega) \cap H_{0}^{1}(\Omega)\right)
$$

for constants $\beta>0, \gamma \geq 0$. Integrating (2.9) in $\tau \in(0, t)$, we have

$$
\begin{aligned}
\int_{0}^{t}\|p\|_{H^{2}(\Omega)}^{2} d \tau & \leq C \int_{0}^{t}\left(\left\|f_{1}\right\|_{L^{2}(\Omega)}^{2}+\|p\|_{L^{2}(\Omega)}^{2}\right) d \tau \\
& \leq C \int_{0}^{t}\left\|f_{1}\right\|_{L^{2}(\Omega)}^{2} d \tau+\|p\|_{L^{\infty}\left(0, T_{e} ; L^{2}(\Omega)\right.}^{2} \int_{0}^{t} d \tau \leq C_{t} .
\end{aligned}
$$

It remains to show that $T_{t} \in L^{2}\left(0, t ; H^{-1}(\Omega)\right)$. To do so, (2.8) is changed to

$$
\left\|T_{t}\right\|_{H^{-1}(\Omega)} \leq C\left(\left\|T_{x x}\right\|_{H^{-1}(\Omega)}+\left\|\frac{\partial h}{\partial p} \frac{\partial p}{\partial t}\right\|\right) .
$$

Thus

$$
\begin{aligned}
\int_{0}^{t}\left\|T_{t}\right\|_{H^{-1}(\Omega)}^{2} d \tau & \leq C \int_{0}^{t}\left(\left\|T_{x x}\right\|_{H^{-1}(\Omega)}^{2}+\left\|\frac{\partial h}{\partial p} \frac{\partial p}{\partial t}\right\|^{2}\right) d \tau \\
& \leq C\left(\left\|T_{x x}\right\|_{L^{2}\left(0, t, H^{-1}(\Omega)\right)}^{2}+\left\|\frac{\partial h}{\partial p}\right\|_{L^{\infty}\left(0, t ; L^{\infty}(\Omega)\right)}^{2}\left\|p_{t}\right\|_{L^{2}\left(0, t ; L^{2}(\Omega)\right)}^{2}\right. \\
& \leq C
\end{aligned}
$$

and $T_{t}$ is bounded in $L^{2}\left(0, t ; H^{-1}(\Omega)\right)$.

We have the solution $p, T$ of (1.8), (1.9). This allows us to extend the solution $p, T$ stepby-step to all of $T_{e}$.

Theorem 2.1 (Uniqueness) Assume that $p$ and $T$ are the weak solution of (1.8)-(1.9). Then the weak solution is unique. 
Proof If $\widetilde{p}, \widetilde{T}$ are another solution, write $w_{1}:=p-\widetilde{p}, w_{2}:=T-\widetilde{T}$. Setting $\varphi=w_{1}$ in (1.16), $\varphi=w_{2}$ in (1.17) and integrating by parts, by using Young's inequality, we have

$$
\begin{aligned}
& \int_{0}^{T_{e}} \frac{d}{d t}\left\|w_{1}(\tau)\right\|^{2} d \tau+\int_{0}^{T_{e}}\left\|w_{1 x}\right\|^{2} d \tau+\int_{0}^{T_{e}} \int_{\Omega} w_{1}^{2}\left(p^{2}+\tilde{p}^{2}\right) d x d \tau \\
& \quad \leq C \int_{0}^{T_{e}}\left\|w_{1}\right\|^{2} d \tau, \\
& \int_{0}^{T_{e}} \frac{d}{d t}\left\|w_{2}(\tau)\right\|^{2} d \tau+\int_{0}^{T_{e}}\left\|w_{2 x}\right\|^{2} d \tau \leq 0 .
\end{aligned}
$$

(2.10) is changed to

$$
\left\|w_{1}\right\|^{2} \leq C \int_{0}^{T_{e}}\left\|w_{1}\right\|^{2} d \tau
$$

By using of Gronwall's inequality, we obtain $p=\widetilde{p}$ for almost everywhere $Q_{T_{e}}$.

(2.11) is changed to

$$
\left\|w_{2}\right\|^{2} \leq 0
$$

we obtain $T=\widetilde{T}$ for almost everywhere $Q_{T_{e}}$.

\section{Regularity}

In this section we discuss the regularity of the weak solutions $p, T$ to the initial-boundary value problem for parabolic-parabolic systems in Sect. 1. Assume that all conditions in Sect. 1 are met and $p_{0} \in H^{2}(\Omega), T_{0} \in H_{0}^{1}(\Omega)$. For this initial $p_{0} \in H^{2}(\Omega), T_{0} \in H_{0}^{1}(\Omega)$, solutions $p, T$ can be constructed as in Sect. 2. Our eventual goal is to prove that $p, T$ is smooth.

Lemma 3.1 There holds, for any $t \in\left[0, T_{e}\right]$,

$$
\begin{aligned}
& \|p\|_{L^{\infty}\left(0, T_{e} ; H^{2}(\Omega)\right)}+\left\|p_{t}\right\|_{L^{\infty}\left(0, T_{e} ; L^{2}(\Omega)\right)}+\left\|p_{t}\right\|_{L^{2}\left(0, T_{e} ; H_{0}^{1}(\Omega)\right)}+\left\|p_{t t}\right\|_{L^{2}\left(0, T_{e} ; H^{-1}(\Omega)\right)} \\
& \quad+\|T\|_{L^{\infty}\left(0, T_{e} ; H_{0}^{1}(\Omega)\right)}+\|T\|_{L^{2}\left(0, T_{e} ; H^{2}(\Omega)\right)}+\|T\|_{L^{2}\left(0, T_{e} ; L^{2}(\Omega)\right)} \\
& \leq\left(C+\left\|p_{0}\right\|_{H^{2}(\Omega)}+\left\|T_{0}\right\|_{H_{0}^{1}(\Omega)}\right) .
\end{aligned}
$$

Proof Differentiating (1.8) formally with respect to $t$ yields

$$
\begin{aligned}
p_{t t}= & \frac{1}{\tau_{12}}\left(\varepsilon_{12}^{2} p_{x x t}-\frac{1}{2 a_{12}}\left(1-6 p+6 p^{2}\right) p_{t}+\frac{m_{12}}{a_{12}}(1-2 p) p_{t}\right) \\
& -\kappa T \frac{\partial^{2} h}{\partial p^{2}} p_{t}-\kappa T_{t} \frac{\partial h}{\partial p}, \\
T_{t}= & D T_{x x}+\frac{1}{2} \frac{\partial h}{\partial p} \frac{\partial p}{\partial t} .
\end{aligned}
$$

Multiplying (3.3) by $-T_{x x}$ and integrating by parts with respect to $x$ over $\Omega$, we have

$$
\frac{1}{2} \frac{d}{d t}\left\|T_{x}\right\|^{2}+D\left\|T_{x x}\right\|^{2}=-\frac{1}{2} \int_{\Omega} T_{x x} \frac{\partial h}{\partial p} p_{t} d x \leq \varepsilon\left\|T_{x x}\right\|^{2}+c_{\varepsilon}\left\|\frac{\partial h}{\partial p}\right\|_{L^{\infty}(\Omega)}^{2}\left\|p_{t}\right\|^{2} .
$$


Thus

$$
\frac{1}{2} \frac{d}{d t}\left\|T_{x}\right\|^{2}+(D-\varepsilon)\left\|T_{x x}\right\|^{2} \leq c_{\varepsilon}\left\|\frac{\partial h}{\partial p_{1}}\right\|_{L^{\infty}(\Omega)}^{2}\left\|p_{t}\right\|^{2}
$$

Integrating (3.4) in $\tau \in(0, t)$, we have

$$
\left\|T_{x}\right\|^{2}+2(D-\varepsilon) \int_{0}^{t}\left\|T_{x x}\right\|^{2} d \tau \leq\left\|T_{0 x}\right\|^{2}+C \leq C .
$$

(3.3) be changed to

$$
D T_{x x}=T_{t}-\frac{1}{2} \frac{\partial h}{\partial p} \frac{\partial p}{\partial t}=f_{3} .
$$

We use the result of regularity theory of elliptic equations

$$
\|T\|_{H^{2}(\Omega)} \leq C\left(\left\|f_{3}\right\|+\|T\|\right)
$$

Squaring and integrating (3.6) in $\tau \in(0, t)$, we have

$$
\begin{aligned}
\int_{0}^{T_{e}}\|T\|_{H^{2}(\Omega)}^{2} d \tau & \leq C \int_{0}^{T_{e}}\left(\left\|f_{3}\right\|^{2}+\|T\|^{2}\right) d \tau \\
& \leq C\left(C+\|T\|_{L^{\infty}\left(0, T_{e} ; L^{2}(\Omega)\right)}^{2} \int_{o}^{T_{e}} d \tau\right) \leq C,
\end{aligned}
$$

we obtain $T \in L^{2}\left(0, T_{e} ; H^{2}(\Omega)\right)$.

Squaring and integrating (3.3) in $\Omega$, we have

$$
\left\|T_{t}\right\|^{2} \leq C\left\|T_{x x}\right\|^{2}+C\left\|\frac{\partial h}{\partial p}\right\|_{L^{\infty}(\Omega)}^{2}\left\|p_{t}\right\|^{2}
$$

Integrating (3.8) in $\tau \in(0, t)$, we have

$$
\int_{0}^{t}\left\|T_{t}\right\|^{2} d \tau \leq C \int_{0}^{t}\left\|T_{x x}\right\|^{2} d \tau+C \int_{0}^{t}\left\|\frac{\partial h}{\partial p}\right\|_{L^{\infty}(\Omega)}^{2}\left\|p_{t}\right\|^{2} d \tau \leq C,
$$

we obtain $T_{t} \in L^{2}\left(Q_{T_{e}}\right)$.

Multiplying (3.2) by $p_{t}$ and integrating by parts with respect to $x$ over $\Omega$, we have

$$
\begin{aligned}
& \frac{1}{2} \frac{d}{d t}\left\|p_{t}\right\|^{2}+\frac{1}{2 \tau_{12} a_{12}} \int_{\Omega}\left(1-6 p+6 p^{2}\right) p_{t}^{2} d x \\
& \quad+\frac{\varepsilon_{12}^{2}}{\tau_{12}}\left\|p_{x t}\right\|^{2}-\frac{m_{12}}{\tau_{12} a_{12}} \int_{\Omega}(1-2 p)\left(p_{t}\right)^{2} d x \\
& \leq C\left\|\frac{\partial^{2} h}{\partial p^{2}}\right\|_{L^{\infty}(\Omega)}\|T\|_{L^{\infty}(\Omega)}\left\|p_{t}\right\|^{2}+\frac{1}{2}\left\|\frac{\partial h}{\partial p}\right\|_{L^{\infty}(\Omega)}^{2}\left\|T_{t}\right\|^{2}+\frac{1}{2}\left\|p_{t}\right\|^{2} \\
& \leq C\left\|p_{t}\right\|^{2}+C\left\|T_{t}\right\|^{2} .
\end{aligned}
$$


Thus

$$
\frac{1}{2} \frac{d}{d t}\left\|p_{t}\right\|^{2}+\frac{\varepsilon_{12}^{2}}{2 \tau_{12}}\left\|p_{x t}\right\|^{2}+c \int_{\Omega} p^{2}\left(p_{t}\right)^{2} d x \leq C\left\|p_{t}\right\|^{2}+C\left\|T_{t}\right\|^{2}
$$

We thereupon conclude from (3.10) that

$$
\begin{aligned}
& \left\|p_{t}(t)\right\|^{2}+\int_{0}^{T_{e}}\left\|p_{x t}\right\|^{2} d \tau+c \int_{0}^{T_{e}} \int_{\Omega} p^{2} p_{1 t}^{2} d x d \tau \\
& \leq e^{C t}\left(\left\|p_{0 t}\right\|^{2}+C \int_{0}^{T_{e}}\left\|T_{t}\right\|^{2} d \tau\right) \leq C\left(\left\|p_{0}\right\|_{H^{2}(\Omega)}^{2}+C\right) .
\end{aligned}
$$

Multiplying (1.8) by $p_{x x}$ and integrating it in $x \in \Omega$, we have

$$
\begin{aligned}
\frac{1}{\tau_{12}} \varepsilon_{12}^{2}\left(p_{x x}, p_{x x}\right)= & \left(p_{t}+\kappa T \frac{\partial h}{\partial p}, p_{x x}\right) \\
& -\frac{1}{\tau_{12}}\left(-\frac{1}{2 a_{12}} p(1-p)(1-2 p)+\frac{m_{12}}{a_{12}} p(1-p), p_{x x}\right) \\
= & \left(g_{3}+p_{t}, p_{x x}\right) .
\end{aligned}
$$

Next we invoke the inequality

$$
\beta\|p\|_{H^{2}(\Omega)}^{2} \leq\left(g_{3}+p_{t}, p_{x x}\right)+\gamma\|p\|^{2}, \quad\left(p \in H^{2}(\Omega) \cap H_{0}^{1}(\Omega)\right)
$$

for constants $\beta>0, \gamma \geq 0$.

We thereupon conclude from (3.12) that

$$
\|p\|_{H^{2}(\Omega)} \leq C\left(\left\|g_{3}\right\|+\left\|p_{t}\right\|+\|p\|\right) \leq C
$$

we obtain $p \in L^{\infty}\left(0, T_{e} ; H^{2}(\Omega)\right)$.

It remains to show that $p_{t t} \in L^{2}\left(0, T_{e} ; H^{-1}(\Omega)\right)$. To do so, equation (3.2) is changed to

$$
p_{t t}=\frac{1}{\tau_{12}}\left(\varepsilon_{12}^{2} p_{x x t}-\frac{1}{2 a_{12}}\left(1-6 p+6 p^{2}\right) p_{t}+\frac{m_{12}}{a_{12}}(1-2 p) p_{t}\right)-\kappa T \frac{\partial^{2} h}{\partial p^{2}} p_{t}-\kappa T_{t} \frac{\partial h}{\partial p}
$$

Thus

$$
\left\|p_{t t}\right\|_{H^{-1}(\Omega)} \leq C\left(\left\|p_{x x t}\right\|_{H^{-1}(\Omega)}+\left\|p_{t}\right\|_{L^{2}(\Omega)}+\left\|T_{t}\right\|_{L^{2}(\Omega)}\right)
$$

and so $p_{t t}$ is bounded in $L^{2}\left(0, T_{e} ; H^{-1}(\Omega)\right)$.

\section{Global attractor}

In this section we discuss the existence of a global attractor and the stability of solution to problem (1.8)-(1.13). This amounts to proving that the solutions of the evolution problem remain bounded as $t \rightarrow \infty$. Usually, proving the existence of absorbing sets amounts to proving a priori estimates. Once the properties of the semigroup are established, we may apply the general results of the attractor. That theorem produces the existence of an attractor which is maximal among the bounded attractors and among the bounded functional invariant sets; it fully describes the long-time behavior of the solutions of the equations. 


\subsection{Global attractor}

Let

$$
g_{1}(p)=\frac{1}{2 \tau_{12} a_{12}} p(1-p)(1-2 p)-\frac{1}{\tau_{12} m_{12}} p(1-p)
$$

be a polynomial with a positive leading coefficient. Using Young's inequality, we infer from (4.1) the existence of a constant $c_{1}>0$ such that

$$
\left|\frac{1}{2 \tau_{12} a_{12}} p\left(1-3 p^{2}\right)-\frac{1}{\tau_{12} m_{12}} p(1-p)\right| \leq \frac{1}{2 \tau_{12} a_{12}} p^{4}+c_{1}
$$

and hence

$$
-\frac{1}{2 \tau_{12} a_{12}} p^{4}-c_{1} \leq g_{1}(p) p \leq \frac{1}{2 \tau_{12} a_{12}} p^{4}+c_{1} .
$$

Lemma 4.1 (The uniform Gronwall Lemma) Let $g, h, y$ be three positive locally integral functions on $\left(t_{0}, \infty\right)$ such that $y^{\prime}$ is locally integrable on $\left(t_{0}, \infty\right)$, and which satisfies

$$
\begin{aligned}
& \frac{d y}{d t} \leq g y+h \quad \text { for } t \geq t_{0}, \\
& \int_{t}^{t+r} g(s) d s \leq a_{1}, \quad \int_{t}^{t+r} h(s) d s \leq a_{2}, \\
& \int_{t}^{t+r} y(s) d s \leq a_{3} \quad \text { for } t \geq t_{0},
\end{aligned}
$$

where $r, a_{1}, a_{2}, a_{3}$ are positive constants. Then

$$
y(t+r) \leq\left(\frac{a_{3}}{r}+a_{2}\right) e^{a_{1}}, \quad \forall t \geq t_{0} .
$$

Proof of Theorem 1.3 (a) Absorbing set in $L^{2}(\Omega)$ of $p$. Using relation (4.3), we obtain, multiplying (1.8) by $p$ and integrating by parts with respect to $x$ over $\Omega$, where we take the boundary condition (1.10) into account, that for almost all $t$

$$
\begin{aligned}
& \frac{1}{2} \frac{d}{d t}\|p\|^{2}+\frac{\varepsilon_{12}^{2}}{\tau_{12}}\left\|p_{x}\right\|^{2}+\frac{1}{2 \tau_{12} a_{12}}\|p\|^{4} \\
& \leq \kappa\|T\|\|p\|_{L^{2}(\Omega)}\left\|\frac{\partial h}{\partial p}\right\|_{L^{\infty}(\Omega)}+2 c_{1}|\Omega| \\
& \leq c_{\varepsilon}\|T\|^{2}\left\|\frac{\partial h}{\partial p}\right\|_{L^{\infty}(\Omega)}^{2}+\varepsilon\|p\|^{2}+2 c_{1}|\Omega|,
\end{aligned}
$$

$|\Omega|=$ the measure (volume) of $\Omega$. Due to Poincarés inequality, there exists a constant $c_{0}=c_{0}(\Omega)$ such that

$$
\|p\| \leq c_{0}\left\|p_{x}\right\|, \quad \forall p \in H_{0}^{1}(\Omega),
$$


and setting $c_{2}=4 c_{1}|\Omega|$, we infer from (4.4) that

$$
\frac{d}{d t}\|p\|^{2}+\left(\frac{2 \varepsilon_{12}^{2}}{c_{0}^{2} \tau_{12}}-2 \varepsilon\right)\|p\|^{2}+\frac{1}{\tau_{12} a_{12}}\|p\|^{4} \leq c_{2}+2 c_{\varepsilon}\|T\|^{2}\left\|\frac{\partial h}{\partial p}\right\|_{L^{\infty}(\Omega)}^{2},
$$

where $\epsilon=\frac{2 \varepsilon_{12}^{2}}{c_{0}^{2} \tau_{12}}-2 \varepsilon>0$.

Using the uniform Gronwall Lemma 4.1 we see that

$$
\begin{aligned}
\|p(t)\|^{2} & \leq\left\|p_{0}\right\|^{2} e^{-\epsilon t}+e^{-\epsilon t} \int_{0}^{t} e^{\epsilon \tau}\left(c_{2}+2 c_{\varepsilon}\|T\|^{2}\left\|\frac{\partial h}{\partial p}\right\|_{L^{\infty}(\Omega)}^{2}\right) d \tau \\
& \leq\left\|p_{0}\right\|^{2} e^{-\epsilon t}+e^{-\epsilon t} \int_{0}^{t} e^{\epsilon \tau}\left(c_{2}+2 c_{3} c_{\varepsilon}\right) d \tau \\
& \leq\left\|p_{0}\right\|^{2} e^{-\epsilon t}+\frac{c_{2}+2 c_{3} c_{\varepsilon}}{\epsilon}\left(1-e^{-\epsilon t}\right) .
\end{aligned}
$$

Thus

$$
\limsup _{t \rightarrow \infty}\|p(t)\| \leq \rho_{0}, \quad \rho_{0}^{2}=\frac{c_{2}+2 c_{3} c_{\varepsilon}}{\epsilon} .
$$

There exists an absorbing set $\mathcal{B}_{0}$ in $L^{2}(\Omega)$, namely, any ball of $L^{2}(\Omega)$ centered at 0 of radius $\rho_{0}^{\prime}>\rho_{0}$. If $\mathcal{B}$ is a bounded set of $L^{2}(\Omega)$, included in a ball $B(0, R)$ of $L^{2}(\Omega)$ centered at 0 of radius $R$, then $S(t) B \subset B\left(0, \rho_{0}^{\prime}\right)$ for $t \geq t_{0}\left(\mathscr{B} ; \rho_{0}^{\prime}\right)$

$$
t_{0}=\frac{1}{\epsilon} \log \frac{R^{2}}{\left(\rho_{0}^{\prime}\right)^{2}-\rho_{0}^{2}}
$$

We also infer from (4.4), after integration in $t$, that

$$
\begin{aligned}
& \int_{t}^{t+r} \frac{2 \varepsilon_{12}^{2}}{\tau_{12}}\left\|p_{x}\right\|^{2} d \tau+\frac{1}{\tau_{12} a_{12}} \int_{t}^{t+r}\|p\|^{4} d \tau \\
& \quad \leq c_{2} r+2 \varepsilon \int_{t}^{t+r}\|p(\tau)\|^{2} d \tau+r c_{3} c_{\varepsilon}+\|p(t)\|^{2}, \quad r>0 .
\end{aligned}
$$

With (4.6) we conclude that

$$
\begin{aligned}
& \limsup _{t \rightarrow \infty}\left(2 \epsilon \int_{t}^{t+r}\left\|p_{x}\right\|^{2} d \tau+\frac{1}{\tau_{12} a_{12}} \int_{t}^{t+r}\|p\|^{4} d \tau\right) \\
& \leq\left(c_{2}+c_{3} c_{\varepsilon}\right) r+(2 \varepsilon r+1) \rho_{0}^{2}, \quad r>0,
\end{aligned}
$$

and if $p_{0} \in \mathscr{B} \subset B(0, R)$ and $t \geq t_{0}\left(\mathscr{B}, \rho_{0}^{\prime}\right)$, then

$$
\begin{gathered}
2 \epsilon \int_{t}^{t+r}\left\|p_{x}\right\|^{2} d \tau+\frac{2}{2 \tau_{12} a_{12}} \int_{t}^{t+r}\|p\|^{4} d \tau \\
\leq\left(c_{2}+c_{3} c_{\varepsilon}\right) r+(2 \varepsilon r+1)\left(\rho_{0}^{\prime}\right)^{2}, \quad r>0 .
\end{gathered}
$$

(b) Absorbing set in $H_{0}^{1}(\Omega)$ of $p$. We now prove the existence of an absorbing set in $H_{0}^{1}(\Omega)$ and the uniform compactness of $S(t)$. For that purpose we need another energytype equality; it is obtained by multiplying (1.8) by $-p_{x x}$ and integrating by parts with 
respect to $x$ over $\Omega$, where we take the boundary condition (1.10) into account, that for almost all $t$

$$
\frac{1}{2} \frac{d}{d t}\left\|p_{x}\right\|^{2}+\frac{\varepsilon_{12}^{2}}{\tau_{12}}\left\|p_{x x}\right\|^{2}+c \int_{\Omega}\left(p^{2}-1\right)\left(p_{x}\right)^{2} d x=\kappa \int_{\Omega} T p_{x x} \frac{\partial h}{\partial p} d x
$$

As in (4.3), we can prove with repeated applications of Young's inequality that there exists $c_{2}^{\prime}>0$ such that

$$
\frac{3}{4 \tau_{12} a_{12}} p^{2}-c_{2}^{\prime} \leq \frac{d g_{1}}{d p} \leq \frac{9}{4 \tau_{12} a_{12}} p^{2}+c_{2}^{\prime}, \quad \forall p \in R
$$

We also infer from general results on the Dirichlet problem in $\Omega$ that $|\triangle p|$ is, on $H_{0}^{1}(\Omega) \cap$ $H^{2}(\Omega)$, a norm equivalent to that induced by $H^{2}(\Omega)$.

Setting $c_{3}^{\prime}=\frac{3}{4 \tau_{12} a_{12}}>0$, we then deduce from (4.12)

$$
\frac{d}{d t}\left\|p_{x}\right\|^{2}+\epsilon^{\prime}\left\|p_{x}\right\|^{2}+c_{3}^{\prime} \int_{\Omega}(p)^{2}\left(p_{x}\right)^{2} d x \leq 2 c_{\varepsilon}\|T\|^{2}\left\|\frac{\partial h}{\partial p}\right\|_{L^{\infty}(\Omega)}^{2},
$$

where $\epsilon^{\prime}=\frac{\frac{2 \varepsilon_{12}^{2}}{\tau_{12}}-2 \varepsilon}{c_{0}^{\prime 2}}-2 c_{2}^{\prime}>0$. If $p_{0} \in H_{0}^{1}(\Omega)$, then the uniform Gronwall Lemma 4.1 shows that

$$
\left\|p_{x}(t)\right\|^{2} \leq\left\|p_{0}\right\|_{H_{0}^{1}(\Omega)}^{2} e^{-\epsilon^{\prime} t}+\frac{2 c_{3} c_{\varepsilon}}{\epsilon^{\prime}}\left(1-e^{-\epsilon^{\prime} t}\right), \quad t>0 .
$$

A bound valid for all $t \in R^{+}$is obtained by application of the uniform Gronwall lemma; for arbitrary fixed $r>0$, we write (4.13)

$$
\frac{d}{d t}\left\|p_{x}\right\|^{2} \leq 2 c_{2}^{\prime}\left\|p_{x}\right\|^{2}+2 c_{\varepsilon}\|T\|^{2}\left\|\frac{\partial h}{\partial p}\right\|_{L^{\infty}(\Omega)}^{2} .
$$

Multiplying by $e^{-2 c_{2}^{\prime} t}$, we obtain the relation

$$
\frac{d}{d t}\left(e^{-2 c_{2}^{\prime} t}\left\|p_{x}\right\|^{2}\right) \leq 2 e^{-2 c_{2}^{\prime} t} c_{\varepsilon}\|T\|^{2}\left\|\frac{\partial h}{\partial p}\right\|_{L^{\infty}(\Omega)}^{2} \leq 2 c_{\varepsilon}\|T\|^{2}\left\|\frac{\partial h}{\partial p}\right\|_{L^{\infty}(\Omega)}^{2} .
$$

Then, by integration between $t$ and $t+r$, we have

$$
\left\|p_{x}(t+r)\right\|^{2} \leq\left(\frac{\int_{t}^{t+r}\left\|p_{x}(t)\right\|^{2} d \tau}{r}+a_{2}\right) e^{a_{1}} \leq\left(\frac{a_{3}}{r}+a_{2}\right) e^{a_{1}}, \quad t \geq t_{*},
$$

provided

$$
\int_{t}^{t+r}\left\|p_{x}(t)\right\|^{2} d \tau \leq a_{3}, \quad a_{1}=2 c_{2}^{\prime} r, \quad a_{2}=2 c_{\varepsilon} c_{3} r, \quad t \leq t_{*} .
$$

An explicit value of $a_{3}$ can be derived from (4.4) and the computation above when $t_{*}=0$. Hence (4.15) provides a uniform bound for $p_{x}, t>r$, while (4.13) provides a uniform bound 
for $p_{x}$ for $0<t<r$. For our purpose, it is simpler and sufficient to set $t_{*}=t_{0}$, in which case, the value of $a_{3}$ is given by (4.10),

$$
a_{3}=\frac{\left(c_{2}+c_{3} c_{\varepsilon}\right) r+(2 \varepsilon r+1)\left(\rho_{0}^{\prime}\right)^{2}}{2 \epsilon} .
$$

It follows that the ball of $H_{0}^{1}(\Omega)$ centered at 0 of radius $\rho_{1}$ is absorbing in $H_{0}^{1}(\Omega)$, when

$$
\rho_{1}^{2}=\left(\frac{a_{3}}{r}+a_{2}\right) e^{a_{1}}
$$

and if $p_{0}$ belongs to the ball $B\left(0, R^{\prime}\right)$ of $H_{0}^{1}(\Omega)$ centered at 0 of radius $r$, then $p(t)$ enters this absorbing set denoted by $\mathcal{B}_{1}$ at a time $t \leq t_{0}+r$ and remains in it for $t \geq t_{0}+r$. At the same time, this result provides the uniform compactness of $S(t)$ : any bounded set $\mathscr{B}$ of $L^{2}(\Omega)$ is included in such a ball $B\left(0, R^{\prime}\right)$, and for $p_{0} \in \mathscr{B}$ and $\geq t_{0}+r, t_{0}, r$ as above, $p(t)$ belongs to $\mathscr{B}_{1}$ which is bounded in $H_{0}^{1}(\Omega)$ and relatively compact in $L^{2}(\Omega)$.

(c) Absorbing set in $L^{2}(\Omega)$ of $T$. Making use of relations (4.10) and (4.15), we obtain

$$
\begin{aligned}
& \left\|p_{x}(t+r)\right\|^{2}+\left(\frac{\varepsilon_{12}^{2}}{\tau_{12}}-\varepsilon\right) \int_{t}^{t+r}\left\|p_{x x}\right\|^{2} d \tau+c \int_{t}^{t+r} \int_{\Omega} p^{2}\left(p_{x}\right)^{2} d x d \tau \\
& \leq 2 c_{\varepsilon} \int_{t}^{t+r}\|T\|^{2}\left\|\frac{\partial h}{\partial p}\right\|_{L^{\infty}(\Omega)}^{2} d \tau+c_{3}^{\prime} \int_{t}^{t+r}\left\|p_{x}\right\|^{2} d \tau+\left\|p_{x}(t)\right\|^{2} \\
& \leq 2 c_{3} c_{\varepsilon} r+c_{3}^{\prime} a_{3}+\rho_{0}^{2} .
\end{aligned}
$$

Squaring and integrating (1.8) over $\Omega$, we have

$$
\begin{aligned}
\left\|p_{t}\right\|^{2} \leq & \frac{2 \varepsilon_{12}^{2}}{\tau_{12}}\left\|p_{x x}\right\|^{2}+4 \kappa^{2}\|T\|^{2}\left\|\frac{\partial h}{\partial p}\right\|_{L^{\infty}(\Omega)}^{2} \\
& +C\left(\|p(1-p)(1-2 p)\|^{2}+\|p(1-p)\|^{2}\right) .
\end{aligned}
$$

Integrating (4.17) in $\tau \in(t, t+r)$, we have

$$
\int_{t}^{t+r}\left\|p_{t}\right\|^{2} d \tau \leq \frac{\varepsilon_{12}^{2}}{\tau_{12}} \frac{2 c_{3} c_{\varepsilon} r+c_{3}^{\prime} a_{3}+\rho_{0}^{2}}{\frac{\varepsilon_{12}^{2}}{\tau_{12}}-\varepsilon}+\left(c_{2}+2 c_{3} c_{\varepsilon}\right) r+2(2 \varepsilon r+1) \rho_{0}^{2}+2 c_{1} r+4 c_{3} c_{\varepsilon} r .
$$

We multiply (1.9) by $T$ and integrate by parts with respect to $x$ over $\Omega$, where we take the boundary condition (1.11) into account, that for almost all $t$

$$
\frac{1}{2} \frac{d}{d t}\|T\|^{2}+D\left\|T_{x}\right\|^{2}=\frac{1}{2} \int_{\Omega} T \frac{\partial h}{\partial p} p_{t} d x \leq \varepsilon\|T\|^{2}+c_{\varepsilon}\left\|\frac{\partial h}{\partial p}\right\|_{L^{\infty}(\Omega)}\left\|p_{t}\right\|^{2} .
$$

Due to Poincaré's inequality, there exists a constant $c_{0}=c_{0}(\Omega)$ such that

$$
\|T\| \leq c_{0}\left\|T_{x}\right\|, \quad \forall T \in L^{2}(\Omega) .
$$

We infer from (4.18) that

$$
\frac{1}{2} \frac{d}{d t}\|T\|^{2}+\left(\frac{D}{c_{0}^{2}}-\varepsilon\right)\|T\|^{2} \leq c_{\varepsilon}\left\|\frac{\partial h}{\partial p}\right\|_{L^{\infty}(\Omega)}\left\|p_{t}\right\|^{2} .
$$


Using the uniform Gronwall lemma, we see that

$$
\|T(t)\|^{2} \leq e^{-2\left(\frac{D}{c_{0}^{2}}-\varepsilon\right)}\|T(0)\|^{2}+2 C_{1} c_{\varepsilon} .
$$

Thus

$$
\limsup _{t \rightarrow \infty}\|T\| \leq \rho_{2}, \quad \rho_{2}^{2}=2 C_{1} c_{\varepsilon}
$$

There exists an absorbing set $\mathcal{B}_{2}$ in $L^{2}(\Omega)$, namely, any ball of $L^{2}(\Omega)$ centered at 0 of radius $\rho_{2}^{\prime}>\rho_{2}$. If $\mathcal{B}$ is a bounded set of $L^{2}(\Omega)$, included in a ball $B(0, R)$ of $L^{2}(\Omega)$ centered at 0 of radius $R$, then $S(t) \mathscr{B} \subset B\left(0, \rho_{2}^{\prime}\right)$ for $t \geq t_{0}\left(\mathscr{B} ; \rho_{2}^{\prime}\right)$

$$
t_{1}=\frac{1}{2\left(\frac{D}{c_{0}^{2}}-\varepsilon\right)} \log \frac{R^{2}}{\left(\rho_{2}^{\prime}\right)^{2}-\rho_{2}^{2}} .
$$

We also infer from (4.18), after integration in $t$, that

$$
\begin{aligned}
2 D \int_{t}^{t+r}\left\|T_{x}\right\|^{2} d \tau \leq & \|T(t)\|^{2}+2 \varepsilon \int_{t}^{t+r}\|T(t)\|^{2} d \tau \\
& +2 c_{\varepsilon} \int_{t}^{t+r}\left\|\frac{\partial h}{\partial p}\right\|_{L^{\infty}(\Omega)}\left\|p_{t}\right\|^{2} d \tau, \quad r>0 .
\end{aligned}
$$

With (4.19) we conclude that

$$
\begin{aligned}
& \limsup _{t \rightarrow \infty} 2 D \int_{t}^{t+r}\left\|T_{x}\right\|^{2} d \tau \\
& \leq(1+2 r \varepsilon) \rho_{2}^{2}+2 c_{\varepsilon} \frac{\varepsilon_{12}^{2}}{\tau_{12}} \frac{2 c_{3} c_{\varepsilon} r+c_{3}^{\prime} a_{3}+\rho_{0}^{2}}{\frac{\varepsilon_{12}^{2}}{\tau_{12}}-\varepsilon} \\
& \quad+2 c_{\varepsilon}\left(\left(c_{2}+2 c_{3} c_{\varepsilon}\right) r+2(2 \varepsilon r+1) \rho_{0}^{2}+2 c_{1} r+4 c_{3} c_{\varepsilon} r\right), \quad r>0,
\end{aligned}
$$

and if $T_{0} \in \mathscr{B} \subset B(0, R)$ and $t \geq t_{1}\left(\mathscr{B}, \rho_{2}^{\prime}\right)$, then

$$
\begin{aligned}
& 2 D \int_{t}^{t+r}\left\|T_{x}\right\|^{2} d \tau \\
& \leq(1+2 r \varepsilon)\left(\rho_{2}^{\prime}\right)^{2}+2 c_{\varepsilon} \frac{\varepsilon_{12}^{2}}{\tau_{12}} \frac{2 c_{3} c_{\varepsilon} r+c_{3}^{\prime} a_{3}+\rho_{0}^{2}}{\frac{\varepsilon_{12}^{2}}{\tau_{12}}-\varepsilon} \\
& \quad+2 c_{\varepsilon}\left(\left(c_{2}+2 c_{3} c_{\varepsilon}\right) r+2(2 \varepsilon r+1)\left(\rho_{0}^{\prime}\right)^{2}+2 c_{1} r+4 c_{3} c_{\varepsilon} r\right), \quad r>0 .
\end{aligned}
$$

\subsection{Large-time behavior of the solutions}

Lemma 4.2 Let $f=f(t)$, which satisfies $f \in L^{1}\left(\mathbb{R}^{+}\right), \frac{d f}{d t} \in L^{1}\left(\mathbb{R}^{+}\right)$, and

$$
\begin{aligned}
& \int_{0}^{\infty}|f(t)| d t \leq C, \\
& \int_{0}^{\infty}\left|\frac{d f}{d t}\right| d \tau \leq C .
\end{aligned}
$$


Then

$$
\lim _{t \rightarrow \infty} f(t)=0 .
$$

Theorem 4.1 Let $p=p(t, x), T=T(t, x),\|p\|^{2} \in L^{1}\left(\mathbb{R}^{+}\right), \frac{d}{d t}\|p\|^{2} \in L^{1}\left(\mathbb{R}^{+}\right),\|T\|^{2} \in L^{1}\left(\mathbb{R}^{+}\right)$, $\frac{d}{d t}\|T\|^{2} \in L^{1}\left(\mathbb{R}^{+}\right)$, and

$$
\begin{array}{cl}
\int_{0}^{\infty}\left\|p_{x}\right\|^{2} d t \leq C, & \int_{0}^{\infty}\left|\frac{d}{d t}\left\|p_{x}\right\|^{2}\right| d \tau \leq C, \\
\int_{0}^{\infty}\|T\|^{2} d t \leq C, & \int_{0}^{\infty}\left|\frac{d}{d t}\|T\|^{2}\right| d \tau \leq C .
\end{array}
$$

Then

$$
\lim _{t \rightarrow \infty}\|p\|_{L^{\infty}(\Omega)}=0, \quad \lim _{t \rightarrow \infty}\|T\|^{2}=0 .
$$

Proof Multiplying (1.8) by $p$ and integrating by parts with respect to $x$ over $\Omega$, we have

$$
\frac{1}{2} \frac{d}{d t}\|p\|^{2}+\left(\frac{\varepsilon_{12}^{2}}{\tau_{12}}-\varepsilon-c c_{0}\right)\left\|p_{x}\right\|^{2}+\frac{c}{\tau_{12} a_{12}}\|p\|_{L^{4}(\Omega)}^{4} \leq c_{\varepsilon}\left\|T_{x}\right\|^{2}\left\|\frac{\partial h}{\partial p}\right\|_{L^{\infty}(\Omega)}^{2},
$$

where $\alpha=\frac{\varepsilon_{12}^{2}}{\tau_{12}}-\varepsilon-c c_{0}>0$. Integrating (4.22) in $\tau \in(0, t)$, we have

$$
\|p(t)\|^{2}+\alpha \int_{0}^{t}\left\|p_{x}\right\|^{2} d \tau+\frac{c}{\tau_{12} a_{12}} \int_{0}^{t}\|p\|_{L^{4}(\Omega)}^{4} d \tau \leq\|p(0)\|^{2}+C_{\varepsilon} \int_{0}^{t}\left\|T_{x}\right\|^{2} d \tau \leq C .
$$

Thus we obtain

$$
\begin{aligned}
& \int_{0}^{t}\left\|p_{x}\right\|^{2} d \tau \leq C, \\
& \int_{0}^{t}\|p\|_{L^{4}(\Omega)}^{4} d \tau \leq C .
\end{aligned}
$$

Multiplying (1.8) by $-p_{x x}$ and integrating by parts with respect to $x$ over $\Omega$, we have

$$
\begin{aligned}
& \frac{1}{2} \frac{d}{d t}\left\|p_{x}\right\|^{2}+\left(\frac{\varepsilon_{12}^{2}}{\tau_{12}}-\varepsilon\right)\left\|p_{x x}\right\|^{2}+\frac{1}{\tau_{12} a_{12}} \int_{\Omega} p^{2} p_{x}^{2} d x-c_{2}^{\prime} \int_{\Omega}\left(p_{x}\right)^{2} d x \\
& \quad \leq c_{\varepsilon}\left\|\frac{\partial h}{\partial p}\right\|_{L^{\infty}(\Omega)}^{2}+\left\|T_{x}\right\|^{2},
\end{aligned}
$$

where $\beta=\frac{\varepsilon_{12}^{2}}{\tau_{12}}-\varepsilon>0$. Integrating (4.25) in $\tau \in(0, t)$, using relations (4.23) and (4.24), we have

$$
\begin{aligned}
& \left\|p_{x}\right\|^{2}+\beta \int_{0}^{t}\left\|p_{x x}\right\|^{2} d \tau+\frac{1}{\tau_{12} a_{12}} \int_{0}^{t} \int_{\Omega} p^{2} p_{x}^{2} d x d \tau \\
& \quad \leq\left\|p_{x}(0)\right\|^{2}+c_{2}^{\prime} \int_{0}^{t}\left\|p_{x}\right\|^{2} d x d \tau+C \int_{0}^{t}\left\|T_{x}\right\|^{2} d \tau \leq C .
\end{aligned}
$$


Thus we obtain

$$
\begin{aligned}
& \int_{0}^{t}\left\|p_{x x}\right\|^{2} d \tau \leq C, \\
& \int_{0}^{t} \int_{\Omega} p^{2}\left(p_{x}\right)^{2} d x d \tau \leq C .
\end{aligned}
$$

Multiplying (1.8) by $-p_{x x}$ and integrating by parts with respect to $x$ over $\Omega$, we have

$$
\begin{aligned}
\frac{1}{2} \frac{d}{d t}\left\|p_{x}\right\|^{2}= & -\frac{\varepsilon_{12}^{2}}{\tau_{12}}\left\|p_{x x}\right\|^{2}+\frac{1}{2 a_{12} \tau_{12}} \int_{\Omega} p(1-p)(1-2 p) p_{x x} d x \\
& +\kappa \int_{\Omega} T p_{x x} \frac{\partial h}{\partial p} d x .
\end{aligned}
$$

Taking the absolute value on both sides of equation (4.28) and integrating it in $\tau \in(0, t)$, using relations (4.26), (4.27), we have

$$
\begin{aligned}
\frac{1}{2} \int_{0}^{t}\left|\frac{d}{d t}\left\|p_{x}\right\|^{2}\right| d \tau \leq & \frac{1}{2 a_{12} \tau_{12}} \int_{0}^{t}\left|\int_{\Omega} p(1-p)(1-2 p) p_{x x} d x\right| d \tau \\
& +\frac{\varepsilon_{12}^{2}}{\tau_{12}} \int_{0}^{t}\left\|p_{x x}\right\|^{2} d \tau+\kappa \int_{0}^{t}\left|\int_{\Omega} T p_{x x} \frac{\partial h}{\partial p} d x\right| d \tau \\
\leq & \frac{\varepsilon_{12}^{2}}{\tau_{12}} \int_{0}^{t}\left\|p_{x x}\right\|^{2} d \tau+C \int_{0}^{t}\left(p^{2}\left(p_{x}\right)^{2}+p_{x}^{2}\right) d \tau+c_{\varepsilon} \int_{0}^{t}\left\|p_{x}\right\|^{2} d \tau \\
& +\varepsilon \int_{0}^{t}\left\|T_{x}\right\|^{2}\left\|\frac{\partial h}{\partial p}\right\|_{L^{\infty}(\Omega)}^{2} d \tau \leq C .
\end{aligned}
$$

Thus we obtain

$$
\frac{1}{2} \int_{0}^{t}\left|\frac{d}{d t}\left\|p_{x}\right\|^{2}\right| d \tau \leq C
$$

By use of Lemma 4.2 and relations (4.23), (4.30), we obtain

$$
\lim _{t \rightarrow \infty}\left\|p_{x}\right\|^{2}=0
$$

By use of Poincaré's inequality, since $p \in H_{0}^{1}(\Omega)$, we have

$$
\|p\|_{L^{\infty}(\Omega)} \leq C\left(\int_{\Omega}\left|p_{x}\right|^{2} d x\right)^{\frac{1}{2}} \rightarrow 0 .
$$

Multiplying (1.9) by $T$ and integrating by parts with respect to $x$ over $\Omega$, we have

$$
\frac{1}{2} \frac{d}{d t}\|T\|^{2}+(D-\varepsilon)\left\|T_{x}\right\|^{2} \leq c_{\varepsilon}\left\|\frac{\partial h}{\partial p}\right\|_{L^{\infty}(\Omega)}\left\|p_{t}\right\|^{2},
$$

where $D-\varepsilon>0$. Integrating (4.33) in $\tau \in(0, t)$, we get

$$
\|T\|^{2}+(D-\varepsilon) \int_{0}^{t}\left\|T_{x}\right\|^{2} \leq C .
$$


Multiplying (1.9) by $T$ and integrating by parts with respect to $x$ over $\Omega$, we have

$$
\frac{1}{2} \frac{d}{d t}\|T\|^{2}+\left(\frac{D}{c_{0}^{2}}-\varepsilon\right)\|T\|^{2} \leq c_{\varepsilon}\left\|\frac{\partial h}{\partial p}\right\|_{L^{\infty}(\Omega)}\left\|p_{t}\right\|^{2},
$$

where $\frac{D}{c_{0}^{2}}-\varepsilon>0$. Integrating $(4.35)$ in $\tau \in(0, t)$, we have

$$
\|T\|^{2}+\left(\frac{D}{c_{0}^{2}}-\varepsilon\right) \int_{0}^{t}\|T\|^{2} \leq C .
$$

Taking the absolute value on both sides of equation (4.33) and integrating it in $\tau \in(0, t)$, using relation (4.34), we have

$$
\int_{0}^{t}\left|\frac{1}{2} \frac{d}{d t}\|T\|^{2}\right| d \tau \leq C \int_{0}^{t}\left\|T_{x}\right\|^{2} d \tau+C \int_{0}^{t}\left\|\frac{\partial h}{\partial p}\right\|_{L^{\infty}(\Omega)}\left\|p_{t}\right\|^{2} d \tau \leq C .
$$

Since $\left.T\right|_{\partial \Omega}=0$, we have

$$
\|T\|^{2}=0, \quad t \rightarrow \infty
$$

\section{Conclusion}

With the help of Banach's fixed point theorem, we prove the existence of weak solutions and study the regularity of weak solutions for the phase-field model. Also we study the existence of a global attractor for this simplified model and investigate the large time behavior of weak solutions.

\section{Acknowledgements}

I gratefully acknowledge the help of Professor Peicheng Zhu, he has offered me valuable suggestion in this study. The author would like to deeply thank all the reviewers for their insightful and constructive comments.

\section{Funding}

This article is in part supported by start-up grant for 1000-plan scholar from Shanghai University, P.R. China.

\section{Availability of data and materials}

Data sharing not applicable to this article as no data sets were generated or analyzed during the current study.

\section{Competing interests}

The author declares that there is no conflict of interests regarding the publication of this paper.

\section{Authors' contributions}

The author carried out the paper and drafted the manuscript. The author read and approved the final manuscript.

\section{Publisher's Note}

Springer Nature remains neutral with regard to jurisdictional claims in published maps and institutional affiliations.

Received: 2 August 2018 Accepted: 15 January 2019 Published online: 30 January 2019

\section{References}

1. Feltham, D.: Arctic sea ice reduction: evidence, models and impacts. Philos. Trans. R. Soc. A, Math. Phys. Eng. Sci. (2015). https://doi.org/10.1098/rsta.2014.0171

2. Mernild, S.H., Mote, T.L., Liston, G.E.: Greenland ice sheet surface melt extent and trends: 1960-2010. J. Glaciol. 57(204), 621-627 (2011)

3. Stroeve, J.C., Markus, T., Boisvert, L., Miller, J., Barrett, A.: Changes in Arctic melt season and implications for sea ice loss. Geophys. Res. Lett. 41(4), 1216-1225 (2014)

4. Alber, H.-D., Zhu, P.: Solutions to a model for interface motion by interface. Proc. R. Soc. Edinb. 138A, $923-955$ (2008) 
5. Kobayashi, R.: Modeling and numerical simulations of dendritic crystal growth. Physica D 63, 410-423 (1993)

6. Leppäranta, M.: A review of analytical models of sea-ice growth. Atmos.-Ocean 31(1), 123-138 (1993)

7. Freita, J., Eicken, H.: Meltwater circulation and permeability of Arctic summer sea ice derived from hydrological field experiments. J. Glaciol. 166, 349-358 (2003)

8. Mcguinness, M.J.: Modelling sea ice growth. ANZIAM J. 50, 306-319 (2009)

9. Kawano, Y., Ohashi, T.: A mesoscopic numerical study of sea ice crystal growth and texture development. Cold Reg. Sci. Technol. 57, 39-48 (2009)

10. Notz, D., Grae Worster, M.: A one-dimensional enthalpy model of sea ice. Ann. Glaciol. 44, 123-128 (2006)

Submit your manuscript to a SpringerOpen ${ }^{\circ}$ journal and benefit from:

- Convenient online submission

- Rigorous peer review

Open access: articles freely available online

High visibility within the field

- Retaining the copyright to your article

Submit your next manuscript at $\gg$ springeropen.com 